\title{
Anal Canal Squamous Intraepithelial Neoplasia
}

National Cancer Institute

\section{Source}

National Cancer Institute. Anal Canal Squamous Intraepithelial Neoplasia. NCI Thesaurus. Code C7490.

A precancerous neoplastic intraepithelial process involving the anal canal. When it is clinically noticeable, it may appear as an eczematous or papillary lesion, plaques, or papules. Morphologically, it is characterized by loss of epithelial stratification and nuclear polarity, cytologic atypia, and the presence of mitotic figures. It may be classified as low or high grade. 